\title{
CASE STUDY ON QUALITY OF LIFE OF BREAST AND TONGUE CANCER PATIENTS
}

\author{
Dr. Molly Joy \\ Head, Department of Psychology, \\ KristuJyanti College, Bangalore, India \\ Anna Mariya Vilson \\ II year M.Sc .Psychology \\ KristuJyanti College, India
}

\begin{abstract}
Breast and tongue cancer is been increasingly seen in the middle age people in current decade. The aim of this research study is to understand the difference between the quality of life of breast and tongue cancer patients.. The sample is been collected with from the different palliative care units in northern parts of Kerala. 5 breast cancer patients and 5 tongue cancer patients were studied in detail with their quality of life. The results from the whole sample showed that there are differences and similarities between the samples. The similarities in the cancer patients was both the breast and tongue cancer patients reported about their children settlement issues, fear of chances of relapse. The differences was seen in the breast and tongue cancer patients was, the tongue cancer patients was more worried about their body image issues than the breast cancer patients and tongue cancer patients who are of male gender reported that as they are the head of the household they are so much worried about the financial crisis that is been happening in their family.
\end{abstract}

Keywords: Quality of life, Breast and tongue cancer patients, descriptive case study.

Cite this Article: Dr. Molly Joy and Anna Mariya Vilson, Case Study on Quality of Life of Breast and Tongue Cancer Patients, Journal of Management (JOM), 6 (4), 2019, pp. 5-12.

http://www.iaeme.com/JOM/issues.asp?JType=JOM\&VType=6\&IType=4

\section{INTRODUCTION}

Cancer is not only one disease; however it's a larger cluster of virtually one hundred diseases. Its two main characteristics are uncontrolled growth of the cells in the human body and the ability of these cells to migrate from the original site and spread the distant sites. If the unfold is not controlled, cancer may result in death. Cancer is a genetic term for a large group of diseases that can affect any part of the body. The major causes of cancer are it arises from one 
single cell. The transformation from a normal cell into a tumor cell is a multistage process, typically a progression from a pre-cancerous lesion to malignant tumors (Sasco, 2004).

Tongue Cancer: This is one in all many sorts of oral (mouth) cancers. Like alternative cancers, it happens once cells divide out of management and kind a growth, or tumor. There are two types. One is termed oral tongue cancer. The other happens at the bottom of your tongue, where it connects to your throat. The major symptoms of tongue cancer are it could be a lump or sore on the facet of the tongue that doesn't depart. It may be pinkish-red in color, there will a pain in or near the tongue, there will be changes in voice, like sounding hoarse, there will be trouble in swallowing. The major causes can be the human papilloma viruses (HPV) which cause cancers on the bottom of the tongue. HPV can also infect the sex organ space and cause cervical cancer, penile cancer, and anal cancer. It's the most common sexually transmitted infection. The other possibilities that lead to tongue cancer are: Use of tobacco, Use of alcohol, jagged teeth, Teeth and gums are not taken care, Genes also may play a role in tongue cancer.

Breast cancer: Breast cancer is cancer that develops in breast cells (Holland, Herndon, \& Chun, 2017). Typically, the cancer forms in either the lobules or the ducts of the breast (Holland, Herndon, \& Chun, 2017). The major causes of Breast Cancer can be it occurs when some breast cells begin to grow abnormally. These cells divide more rapidly than healthy cells do and continue to accumulate, forming a lump or mass. Cells may spread (metastasize) through your breast to your lymph nodes or to other parts of your body. Breast cancer most often begins with cells in the milk-producing ducts (invasive ductal carcinoma). It may also begin in the glandular tissue called lobules (invasive lobular carcinoma) or in other cells or tissue within the breast. (Clinic, 2019).

Quality of Life (QOL): It is the final well-being of people and societies, outlining negative and positive features of life. It observes life satisfaction, as well as everything from physical health, family, education, employment, wealth, spiritual beliefs, finance and therefore the setting (Barcaccia, 2013). QOL contains a wide selection of contexts, including the fields of international development, healthcare, politics and employment. It is important not to mix up the concept of QOL with a more recent growing area of health related QOL (HRQOL) (Bottomley, 2002). An assessment of HRQOL is effectively associate in Nursing analysis of QOL and its relationship with health.

Need and significance of the study: The highest number of cancer reported among women is breast cancer and tongue or lung cancer among men. The studies show that after breast cancer treatment like mastectomy and chemotherapy women are becoming more anxious about their appearance and so reduced quality of life. In men, it is the cause for tongue or lung cancer because of tobacco and substance abuse. So, it's been reporting that they are showing the negative emotions like anger, depression, guilty feeling etc., because of their substance abuse behavior. And so there is reduction in their quality of life.

This research majorly focuses on the sample of breast cancer and tongue cancer in the 45 to 52 years old people with cancer among women and men. The selected sample is of the middle aged group, they are defined with the group of family responsibilities. As the sample is selected from a village area, it is more prominent with them. The researcher is finding out the difficulties and disturbing thoughts of the patients with the cancer disease how much it is been affected in their daily life routine and their quality of life. This research is to find out that whether there is any relation and significance with the various aspects of a person like physical aspects, psychological and emotional aspects, and sexual aspects, and financial aspects, social and interpersonal aspects with the quality of life of the patients. 


\section{REVIEW OF LITERATURE}

The summary of the review of literature states that there is an evident difference in the quality of life of the breast cancer and tongue cancer patients. In the breast cancer patients the major issues the studies have stated the mental health domain, decreasing level of social and emotional support, role of chemotherapy and radiations' adverse effect in sleep, negative impact of mastectomy on body image issues among married women, particularly among young married women, physical issues and changes after the treatment. In the tongue cancer patients the major domains commonly affected were the functional, physical and head and neck concerns, limited mouth opening and so limited swallowing domain. The researchers have found that the quality of life of tongue cancer patients is low till eight months to one year and after that on as they are completely recovered from the disease their quality of life is stated very good. Also, studies shows that reconstructive techniques play a crucial role in maintenance of satisfactory quality of life.

Research Gap: The review of literature explains about the researches that have been done on the individual basis in quality of life of the people with breast and tongue cancer. The current research explores about the relation with the quality of life of breast and tongue cancer patients in a case study method. The statistics is showing as breast and tongue cancers are the top two types of cancers affected among people. Both breast cancer and tongue cancer shares a statistics of 2.09 million cases (according to 2018 statistics of world health organization). Expressing concern over these data and the number of breast cancer and tongue cancer that have increased in the northern parts of Kerala, current research explores a case study analysis on breast and tongue cancer patients in northern parts of Kerala.

\section{METHOD}

Research Design: Descriptive case study using qualitative approach.

\subsection{Objectives of the study}

- To analyze the case details of breast and tongue cancer patients

- To study Quality of life of breast and tongue cancer patients

- To understand the variations or differences in the quality of life between the breast and tongue cancer patients

- To analyze factors like psychological, physical, sexual, social, financial and health services they have received to assist their quality of life.

\subsection{Operational definition}

Cancer: Cancer is the uncontrolled growth and spread of cells in the body. It can be affected in any part of the body. The uncontrolled growth can also invades surrounding tissues and can spread to distant sites of the body.

Breast Cancer: Breast cancer is the uncontrolled growth of cyst or tumor in the breast of the person.

Tongue Cancer: Tongue cancer is the uncontrolled growth of cells in the cells of tongue and can cause lesions or tumors in the tongue. Tongue cancer is the type of head and neck cancer.

Quality of life: Quality of life is a highly subjective measure of happiness that is an important component of many financial decisions. Factors that play a role in quality of life vary according to personal preferences, but they often include financial security, job satisfaction, family life, health and safety. 
Descriptive Case study: Descriptive Case study can be defined as the one that is focused and detailed, in which propositions and questions about a phenomenon are carefully scrutinized and articulated at the outset. Each case for the study is been descriptively studied.

Sample and sampling technique: The universe of the current research study is Cancer patients. The geographical area for the current research study is from the rural parts of Northern part of Kerala (From the newspaper articles and oncology reporting surveys it was seen that the most number of cases reported in the rural parts of northern Kerala was Breast and tongue cancer patients among women and men respectively) (Tarver, (2012).). The samples for the current research study are Men and women with tongue and breast cancer who are in the age range of 45 to 52 years. The sample is been selected from the rural parts of northern part of Kerala. Samples have been collected from the palliative units of these villages. The informed consent and abstract of the research was given to the palliative care units heads and clients in prior to the interview.

The sampling technique used for the current research is Purposive sampling technique. The purposive sampling technique is a type of non-probability sampling that is most effective when one needs to study a certain cultural domain with knowledgeable experts within. Purposive sampling may also be used with both qualitative and quantitative research techniques. The sampling distribution of the current research with the inclusion and exclusion criteria include

Inclusion criteria: The inclusion criteria for the current research study are: Married Cancer patients, Women with Breast cancer and Men with Tongue cancer, Age from 45 to 52 years, Cancer patients from Northern part of Kerala, Breast cancer patients who have undergone mastectomy, chemotherapy, radiation, hormone therapy and medication treatment phases, Tongue cancer patients who have undergone surgery, chemotherapy, and radiation therapy and medication treatment phases, Cancer patients who are in their follow-up treatments.

Exclusion criteria: The exclusion criteria of the current research study are: Patients with cancer other than breast and tongue cancer, Patients who are not in follow up stage, Cancer patients from other parts of Kerala, Cancer patients who are in the age of $1-44$ and 52 and above

Research ethics followed: The ethical committee and scientific committee of the different Palliative units studied the synopsis, questionnaire and relevance of the study for both the units and patients and approval was given for the researcher to do the data collection for the research, Informed consent and abstract of the research is given to the sample before collecting the data, Beneficence, justice, and the research participants' autonomy is considered throughout the research, The data that is been collected from the sample will be kept confidential, The information collected will be used only for the purpose of this research study, The participation for the research was self-voluntary, samples were free to quit at any point of time.

Measures used for the study: The current research used a semi-structured questionnaire which is been made by the researcher with the help of psychologists, doctors, nurses, and other health care practitioners. The questions were covering various aspects about the cancer patients. The conditions included the Physical, Psychological or emotional, Financial, Sexual, Interpersonal and social and Health care facilities of the patient. Both English and Malayalam version of questionnaire was prepared according to the convenience of the sample.

Procedure: Participants were seen individually at their houses. The informed consent was taken from the palliative heads of different units and clients. Informed consent was given to the patients prior to the interview. After the clients read through the consent and synopsis of the research, the consent was taken and interview was done. Researcher has made the ethics clear to the clients' once again orally before starting the interview. Participants were given with the option of answering the questions in oral and written form. 5 cases of breast cancer and 5 cases of tongue cancer were studied who were fulfilling all the criteria for the inclusion. The 
demographic details were collected from the samples to frame various dimensions related to the quality of life of the patients. The demographic details included age, sex, educational qualification, religion, annual income, etc,. The details of the cases with their quality of life were collected and finally, the results were analyzed subjectively and find out what all issues they are facing in diagnosis, treatment and post treatment phases.

Case study analysis: Case study analysis is been done for the collected data from the selected sample. A case study is a research strategy and an empirical inquiry that investigates a phenomenon within its real-life context. A case study is a descriptive and exploratory analysis of a person, group or event.

\section{CASE ANALYSIS AND RESULTS}

Ten cases were studied. 5 cases of breast cancer and 5 cases of tongue cancer were descriptively studied.

The breast cancer patients who were interviewed were in the age of from 45 year old to 49 year old. The basic educational qualifications of all the clients were at pre-degree. Three of the clients had completed their degree in different subjects and working as teachers in schools/colleges. One of the clients is working as an insurance dealer and one of the clients is working as housewife. All the breast cancer patients were of more than 4 year survivorship.

The treatment phases that the breast cancer patients have undergone are: Mastectomy, Chemotherapy, Radiation (different patients had different number cycles of chemotherapy and radiation), Hormone therapy, medications and follow up stage. In breast cancer patients, the major issues reported by them on the various factors of quality of life of the persons were, emotional well-being, sexual life, financial stability, social and interpersonal relationships, health services that was available and affordable for them and fear of chances of relapse of the disease.

The tongue cancer patients who were interviewed were in the age of from 47 year old to 52 year old. The basic educational qualifications of all the clients were at SSLC. Four of the clients are working as farmer and one of the clients is working as an NGO social worker.

The treatment phases that the tongue cancer patients have undergone are: Surgery, Chemotherapy, Radiation (different patients had different number cycles of chemotherapy and radiation), immunotherapy, medications and follow up stage. In tongue cancer patients, the major issues reported by them on the various factors of quality of life of the persons were, emotional well-being, financial issues, fear of losing responsibilities that he have to complete, fear of chances of relapse of the disease, physical pain and body image issues.

\section{DISCUSSION}

The results of the current research show that as the survivorship lengthens the overall quality of life of the patients' increases. The social support that is been gets for the patients increase their confidence to engage with the social situations and functions same as before. (Spatuzzi \& Vespa, Evaluation of social support, quality of life and body image in women with breast cancer, 2016) Support from the family members and the care taker increase the patient's selfmotivation to increase and to fight against the disease. The patients' have reported that when they are helped emotionally also along physical treatment, they recover fast than the people with less emotional support. The body image issues were not a problem for the clients as they had so much emotional support from the care taking people and society.

The breast cancer patients have reported that, in the beginning they had physical issues and emotional issues to get coped up with. ( $M \&$ P, 2000). The support from the care taker and family members played a major role for their faster recovery. They also have stated that the 
emotional support helped them not only to recover fast, but not to get into fear that they are important for them, which help them and understand it is just a disease and deal with it. The major issues reported by the breast cancer patients include, the settlement issues for their children, the work stress issues, financial crisis that have happened because of the disease treatment and fear of chances of relapse. The overall level of quality of life that was reported by the breast cancer patients was moderate.

The results from tongue cancer patients reported mild and moderate level of quality of life. ( $S \& \mathrm{~J}, 2017$ ). The major issue that was reported by the patients was, as they are the head of the household more of responsibilities were there for them. The thoughts of responsibilities make them more stressed about their life than of their disease. All the tongue male cancer patients have reported that they have this thought, it is a common thought that all fathers carry. The patient who had tongue cancer which was said to be from the cause of substance abuse, he marked a very low quality of life. He said that he is having so much guilt feeling that because of his behavior of substance he was affected with the disease and if he would have not done that he must be have not affected with the disease.

The tongue cancer patients also stated that both emotional and psychological support is needed for the patients when they are affected with the disease. (A \& J, 2008). The major issues that tongue cancer patients reported include, the head of the house hold issues, thinking about children and their settlement, the financial crisis that have happened because of the treatment and the fear of chances of disease relapse. The overall level of quality of life reported by the tongue cancer patients was moderate level.

Find out the difference between the quality of life of breast and tongue cancer patients.

The data was been collected by the researcher in person to each case. After the

\subsection{Findings of the Study}

Both the breast and tongue cancer patients have reported that they have moderate level of quality of life in overall.

The patients who had more family and social support were able to cope up fast to the disease condition and deal with the situations.

The tongue cancer patient who was a substance abuser had the guilty feeling of because of his action the disease have occurred and if would have not done that he must have not affected with the disease.

The patient who are aware about their unhealthy behavior which lead to the disease is more depressed and been psychologically affected than the other patients who were not substance abusers.

As the cancer patients who are been interviewed is in their middle age, they have reported the motherhood and fatherhood issues, for tongue cancer patients as they are the head of household, they have reported a high level of stress on the financial issues that they are facing and the settlement issues for their children.

It's found that even the people who had not exposed to any substance abusing behavior are also affected with the tongue cancer, so from doctors it is been found that tongue cancer is also reported because of the genetic issues, pollution issues, food habit, etc.,

Body image was not an issue reported by the breast cancer patients, they were sad when they lost their hair during the chemotherapy treatment, but it was not a big issue for them because of the support that they were getting from the care giver.

The comparing results of the breast and tongue cancer patients shows those they less worried about their own self rather they are worried about their children and the financial loss that can happen to their children and family. 
The tongue cancer patients have reported that before the diagnosis of the disease they were more socially active, now because of the scar on their face, they are less motivated to go out of the house that they have of answering to the questions by people.

\section{CONCLUSION}

By using the results of the study the government, health care professionals, insurance agents, can help the cancer patients in various ways. (Defrank, 2007).

By using the results of the study Families can be trained accordingly for the better quality of life of cancer patients. (Spatuzzi, Evaluation of Social Support, Quality of Life, and Body Image in Women with Breast Cancer, 2016).

By using the results of this study, further studies can be done.

For clinical purpose this study could be effective. (Defrank, 2007).

Counselors can assist cancer patients with quality of life difficulties with this information. (Defrank, 2007).

The health sector assistants can give the awareness to the common village people with referring to this research. (Defrank, 2007)

Limitations of the study:

The truthfulness of the results depends on the honesty of the respondents. May be possible that the participants, due to apprehensions of being judged wrongly, responded with ideal' answers.

The population of the study only consisted of rural parts of Northern parts of Kerala; hence it cannot be generalized for all breast and tongue cancer patients around the world.

As it is case study research the response acquired is from limited sample, hence it cannot be generalized to the whole population of breast and tongue cancer patients.

The collected demographic details of the patients are not studied in the research study.

\section{REFERENCES}

[1] Aizer, Marital Status and Survival in Patients with Cancer. J Clin Oncol, 31(31), 2013, pp 3869-3876.

[2] Anand, Cancer is a preventable disease that requires major lifestyle changes. Pharmaceutical Research. 25 (9), 2008, pp 2097-116.

[3] Andrew, the Cancer Patient and Quality of Life. The Oncologist, 7 (2), 2002, pp 120-125

[4] Bottomley. (April 2002). The Cancer Patient and Quality of Life. The Oncologist, 7 (2), 120-125. doi: 10.1634/theoncologist.7-2-120. ISSN 1083-7159. PMID 11961195. Retrieved on November 20, 2018

[5] Garenfski, N. \& Kraaiji, V. K, Sponshoven, P, Negative life events, cognitive emotion regulation problems. Personality and individual differences. 30, 2001, pp 1311-1327.

[6] Giese-Davis, J, Koopman, C, Butler, L. D, Classen, C, Cordova, M, Fobair, P, \& Spiegel, D. (2002). Change in emotion-regulation strategy for women with metastic breast cancer following supportive-expressive group therapy, Journal of consulting and clinical psychology, 70(4), 916.

[7] Gross, J. J, Emotion regulation in adulthood: timing is everything. Current directions in psychological science, 10(6), 2001, pp 214-219.

[8] Rothwell (January 2011). Effect of daily aspirin on long-term risk of death due to cancer: Analysis of individual patient data from randomized trials. Lancet, 377 (9759), 31-doi: 10.1016/S0140-6736(10)62110-1. PMID 21144578. Retrieved on November 20, 2018 
[9] Sasco, Tobacco smoking and cancer: a brief review of recent epidemiological evidence. Lung Cancer. 45 Suppl 2: S3-. Retrieved on November 20, 2018

[10] Spattuzi, R, Evaluation of Social Support, Quality of Life, and Body Image in Women with Breast Cancer. NCBI. doi:10.3897/bdj.4.e7720.figure2f, 2016 